\title{
USO DE SIMULADORES DE DIREÇÃO APLICADO AO PROJETO DE SEGURANÇA VIÁRIA
}

\author{
Use of Driving Simulators Applied to Road Safety Project \\ FELIPE RABAY LUCAS \\ LUIS EDUARDO ABRANTES RUSSO \\ RENATA SAYURI KAWASHIMA \\ AURENICE DA CRUZ FIGUEIRA \\ ANA PAULA C. LAROCCA \\ FELIPE ISSA KABBACH JR. \\ Universidade de São Paulo \\ Departamento de Engenharia de Transportes da Escola Politécnica da USP \\ Caixa Postal 61548 CEP 05508-900 - São Paulo - SP, Brasil \\ aurenice@usp.br; larocca.ana@usp.br
}

\section{RESUMO}

O presente documento apresenta e discute tecnologias de visualização por meio de simuladores de direção e sua aplicação na engenharia de transportes, no que tange o projeto rodoviário e seu auxílio na melhoria da segurança das rodovias. O artigo foi elaborada com vistas a contribuir para o desenvolvimento e modernização tecnológica de parte dos estudos desenvolvidos no Brasil, relacionados com a segurança em rodovias, que se apresentam desatualizados em quase 15 anos em relação ao estado-da-arte desta linha de pesquisa em países pioneiros, como é o caso dos EUA, França, Itália, Alemanha. Esta desatualização culmina por contribuir para um cenário nacional trágico, onde, nos últimos dois anos, morreram 42.844 pessoas decorrentes de acidentes em trânsito - número que colocou as rodovias brasileiras entre as mais perigosas do mundo e o Brasil como $5^{\circ}$ país entre os recordistas em mortes no trânsito. Apresenta inicialmente uma descrição técnica e as potencialidades e limitações dos simuladores de direção na condução de experimentos que apresentam realismo natural, com a vantagem do controle das diversas variáveis do estudo, sem risco aos usuários. Adicionalmente, descreve os principais tipos de simuladores de direção existentes no mercado, com a caracterização dos três níveis de sofisticação - simuladores de nível básico, nível intermediário e alta fidelidade.

Palavras-chave: Simuladores de Direção; Segurança Viária; Projeto Geométrico.

Bol. Ciênc. Geod., sec. Comunicações/Trab. Técnicos, Curitiba, v. 19, no 2, p.341-352, abr-jun, 2013. 


\section{ABSTRACT}

This document presents and discusses visualization technologies through driving simulators and its application in transportation engineering, regarding the road project and their help in improving road safety. The article was prepared aiming at contributing to the development and technological modernization of part of the studies carried out in Brazil, related to safety on highways, which is nearly 15 years outdated compared to state-of-art in this research line by pioneer countries such as the U.S., France, Italy, and Germany. This fact contributed to a national tragic scenario, where, for the past two years, 42,844 people died due to traffic crashes, a number that places the Brazilian roads as the top dangerous in the world and the Brazil as the 5th country among the record holders in traffic deaths. This paper presents a technical description and the potentialities and limitations of driving simulators to conduct experiments that show natural realism, with the advantage of controlling the many study variables, without risk to users. Additionally, it describes the main types of driving simulators in the market, with the characterization of the three levels of sophistication - basic level, intermediate level and high fidelity simulators.

Keywords: Driving Simulators; Roadway Safety; Geometric Design.

\section{INTRODUÇÃo}

A cada dia, um grande número de pessoas ao redor do mundo envolve-se em acidentes de trânsito nas vias urbanas e rurais. Ao mesmo tempo, o aumento do número de veículos nas vias sem a adequada infraestrutura, falta de estudos específicos sobre o comportamento do condutor brasileiro e normas adaptadas ao cenário brasileiro são uma das principais razões para a ocorrência do grande número dos acidentes.

As mortes e lesões pessoais no tráfego têm sido um dos maiores problemas de saúde no mundo. A cada 30 segundos uma pessoa é morta em um acidente de estrada - mais de 3.000 por dia - e quase 1,2 milhões de pessoas por ano. Nove em cada dez mortes ocorrem em países em desenvolvimento, cenário onde o Brasil se encontra.

Apesar de mais de 85 por cento das mortes de trânsito e lesões ocorrerem em países do terceiro mundo, neles se concentram apenas cerca de $40 \%$ de veículos automotivos em uso no mundo.

Estimativas indicam que, ao longo dos próximos 15 anos, o número de pessoas que morrem anualmente em acidentes de trânsito pode aumentar para 2,4 milhões, ocorrendo, notadamente, nos países em desenvolvimento e em transição para o primeiro mundo, a menos que sejam tomadas medidas sérias.

Os usuários em risco são especialmente as crianças - 500 crianças morrem por dia em acidentes de trânsito. Em muitos países Asiáticos, Africanos e países do Oriente Médio, entre 40 e 50 \% de pessoas mortas, em acidentes de trânsito, são pedestres e a morte nas estradas é o segundo fator de causa de morte entre os jovens, 
sendo que a maioria das mortes e lesões poderiam ter sido evitadas (Road Safety on Four Continents Conferences, Comitê, 2013).

Esses números colocam a questão da segurança nas rodovias, como um problema de saúde pública.

Há dois anos, quando a Organização das Nações Unidas (ONU) recomendou aos países membros a adoção de planos estratégicos para reduzir o número de acidentes de trânsito, morreram no Brasil 42.844 pessoas - número que colocou as rodovias brasileiras entre as mais perigosas do mundo. De acordo com o Ministério da Saúde, 45,7\% das vítimas tinham entre 20 e 39 anos (19.580) e mais de $80 \%$ eram homens (34.941) (Câmara do Deputados, divisão Transporte e Trânsito, 2012).

Diante disso, tornam-se necessários estudos envolvendo a identificação das causas dos acidentes em relação a diversos fatores, tanto de natureza humana, quanto aqueles relacionados as características das vias, dos veículos e do ambiente.

Os simuladores de direção oferecem a possibilidade de condução de experimentos, apresentado um realismo natural, com a vantagem do controle das diversas variáveis do estudo, sem risco aos usuários.

A contextualização da importância da visualização 3D e simuladores de direção realísticos, nas pesquisas sobre segurança em rodovias, remete obrigatoriamente aos estudos desenvolvidos pelo Federal Highway Administration dos Estados Unidos em conjunto com o Strategic Highway Research Program (SHRP), do Transportation Research Program (TRB), visando sempre à prevenção, mitigação de acidentes e estudos de cognição datando as primeiras investigações do ano 2000.

Quase 15 anos se passaram desde as primeiras investidas destas Agências de Transportes Americanas na utilização das tecnologias 3D e de simuladores de direção. No Brasil, entretanto, nenhum passo foi dado na direção de usar estas ferramentas para estudo do comportamento do tráfego, notadamente num país com grandes distinções regionais de sua população, em comportamento e cultura.

\section{SIMULADORES DE DIREÇÃO}

Em linhas gerais, um simulador de direção consiste num condutor sentado em um cockpit equipado com controles normais como volante, embreagem, pedais de acelerador e freio que estão interligados e permitem a ele conduzir um veículo por uma rodovia que é projetada à sua frente, em telas. Através de softwares específicos, as ações do condutor e a posição do veículo virtual são continuamente calculadas e registradas.

A visão que o condutor terá da via, de dentro do veículo virtual, é projetada em tempo real, em telas ou monitores. O cenário da rodovia é criado a partir de renderização do seu arquivo de projeto geométrico inserindo elementos desejáveis (placas, árvores, topografia, barreiras de concreto, defensas metálicas, etc).

Há quase 15 anos, os simuladores de direção são utilizados em investigações e pesquisas para desenvolvimento de veículos, treinamento de condutores (de trens, 
aeronaves, veículos militares e mais recentemente de caminhões e veículos de passeio) e também em estudos de comportamento de condutores no tráfego.

Diversas razões justificam o uso de simuladores de direção nos estudos de tráfego: alterações no projeto geométrico e investigação do uso de dispositivos de segurança e sinalização, que ainda não existem e são caros para serem construídos apenas para teste e no auxílio ao estudo do comportamento do usuário na via.

Outra razão, bastante forte, para usar simuladores de direção se apoia no fato de que, nele, as situações potencialmente perigosas podem ser investigadas sem riscos à vida. E os motoristas podem ser repetidamente confrontados com eventos que podem ocorrer raramente na realidade. Assim, os comportamentos em diferentes circunstâncias, incluindo condições climáticas específicas - como neblina, chuva podem ser estudados (BELLA, (2008); BENEDETTO et al., (2002); KLEE et al., (1999); GODLEY et al., (2002); SANTIAGO-CHAPARRO et al., (2011); OLIVER (2012); FISCHER \& ERIKSSON (2012); BHATTI et.al (2012)).

Usualmente, os simuladores são classificados de acordo com a tecnologia empregada e os custos embarcados. Também podem ser classificados pelo tipo de aplicação dado.

O nível de sofisticação e competência no estudo pode apresentar variações em cada simulador. A influência do realismo da simulação e da variedade de simulações recriadas deve ser levada em consideração na escolha e no desenvolvimento do simulador. E para garantir a sua consistência, um cenário deve identificar quais fatores influenciam o comportamento do condutor para replicar as circunstâncias essenciais em cada experimento. Em adição, para prover uma plataforma capaz de examinar como os motoristas respondem em situações críticas, os simuladores oferecem um meio controlado para estudar o desempenho destes. A partir da simulação de cenários onde as propriedades das rodovias e os padrões de tráfego são precisamente controlados, é possível estimar as diversas características normativas do comportamento dos motoristas que são difíceis de analisar a partir dos estudos de observação do mundo real. Dessa forma, as respostas de um condutor podem ser medidas, estimadas e controladas para diversos cenários de investigação em um simulador apropriado.

Os diversos simuladores exploram os principais canais sensoriais que afetam a percepção dos condutores, como o sistema visual, de moção e auditivo (ANDERSEN, 2011). Cada um à sua maneira, eles são capazes de realizar as medições dos diversos fatores que afetam o comportamento e a segurança dos motoristas.

A seguir, apresenta-se uma breve descrição dos diversos tipos de simuladores e considerações sobre suas aplicações.

\subsection{Simuladores de nível básico}

Com relação à segurança dos motoristas, os simuladores de nível básico são amplamente usados nos treinamentos dos condutores, com a exploração básica do sistema sensorial visual e auditivo.

Bol. Ciênc. Geod., sec. Comunicações/Trab. Técnicos Curitiba, v. 19, nº 2, p.341-352, abr-jun, 2013. 
Geralmente, o equipamento é constituído por desktops, com telas sobre as quais são projetados os cenários de tráfego (Figura 1).

Figura 1. Simuladores desktop de nível básico da STISIM. Fonte: http://www. stisimdrive.com.

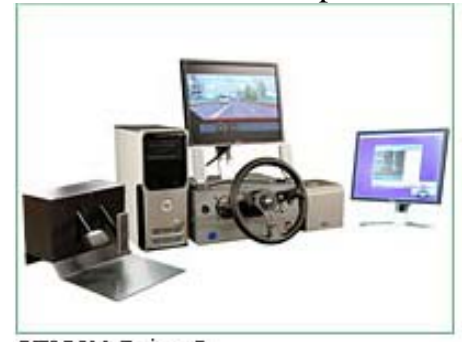

STISIM Drive

Driving Simulator - M300

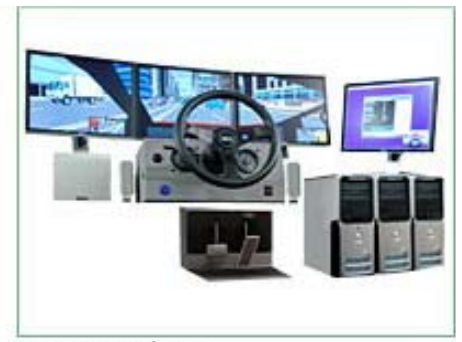

STISIM Drive

Driving Simulator - M400

Com relação as pesquisas de comportamento de direção, Jamson (2011) justifica a aplicação dos simuladores de nível básico em estudos onde a percepção da cena visual é o aspecto chave, a exemplo das pesquisas focadas na alteração do comportamento do condutor por mudança de cena (CHO et al., 2011; HONG et al., 2001).

\subsection{Simuladores de nível intermediário}

Os simuladores de nível intermediário possuem um campo de visão horizontal mais amplo, além de, geralmente, também serem constituídos por um ambiente que representa a cabine de um veículo real. Isto permite uma maior exatidão na medição do comportamento do condutor (Figura 2).

Figura 2. Simulador de nível intermediário.

Fonte: http://www.vi-grade.com/index.php?pagid=drivesim

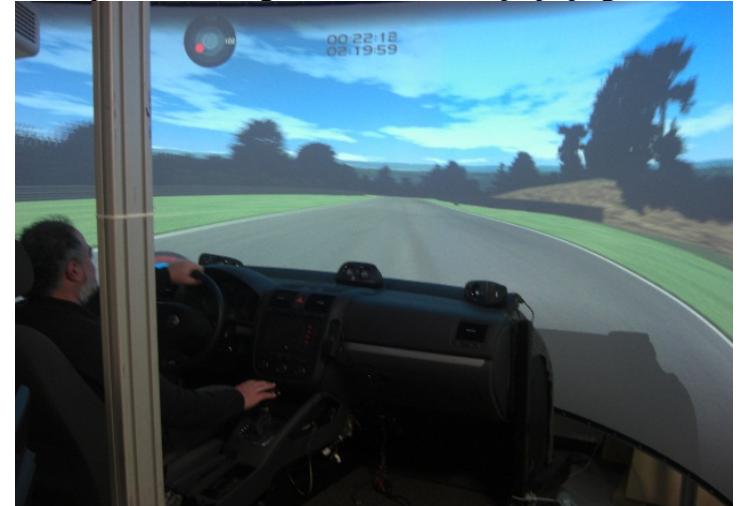

Bol. Ciênc. Geod., sec. Comunicações/Trab. Tecnicos Curitiba, v. 19, nº 2, p.341-352, abr-jun, 2013. 
Este tipo de simulador é utilizado em pesquisas mais avançadas (quando comparadas as pesquisas em simuladores de nível básico), que requerem uma percepção mais acurada do ambiente da rodovia e dos itens que influenciam o comportamento do condutor, como, por exemplo, as sinalizações horizontal e vertical (JAMSON, 2011). Seu uso também é bastante recomendado em pesquisas de comportamento que requerem métricas mais precisas, como por exemplo, a medição do tempo de reação para frenagem. (ALLEN et al, 2008).

A Figura 3, a seguir, ilustra um exemplo de aplicação do simulador de nível intermediário. No caso representado, foi estudado o comportamento dos condutores em relação aos elementos de sinalização indicativos do início da faixa reversível, visando verificar se os dispositivos instalados na via permitem que o condutor realize a manobra de mudança de faixa de forma segura.

Figura 3. Aplicação de simulador de nível intermediário - Percepção da sinalização indicativa de faixa reversível.

Fonte: http://www.its.leeds.ac.uk.

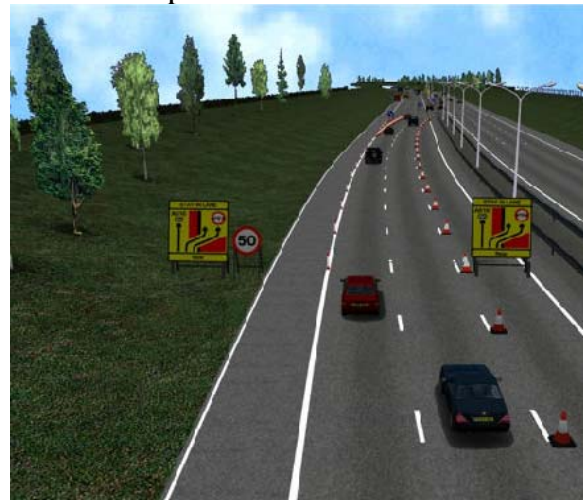

\subsection{Simuladores de alta fidelidade}

Nos simuladores de alta fidelidade, a exploração dos sentidos, principalmente de movimento, é feita de forma a recriar o ambiente de tráfego da maneira mais próxima possível do real, sendo capaz de reproduzir com precisão sinais de movimento de aceleração sustentada, manobras de frenagem, movimento em múltiplas faixas de tráfego e interação com as diferentes superfícies da estrada.

Este tipo de equipamento, dotado de sistemas de movimentação de alta qualidade, torna possível a simulação de manobras e situações limite específicas, como uma mudança repentina de faixa ou frenagem de emergência, sem oferecer risco de lesões ao condutor (Figura 4), ao contrário do que poderia ocorrer em um teste real de campo. 
Figura 4. Simulador de alta fidelidade. Fonte: http://www.nads-sc.iuowa.edu.

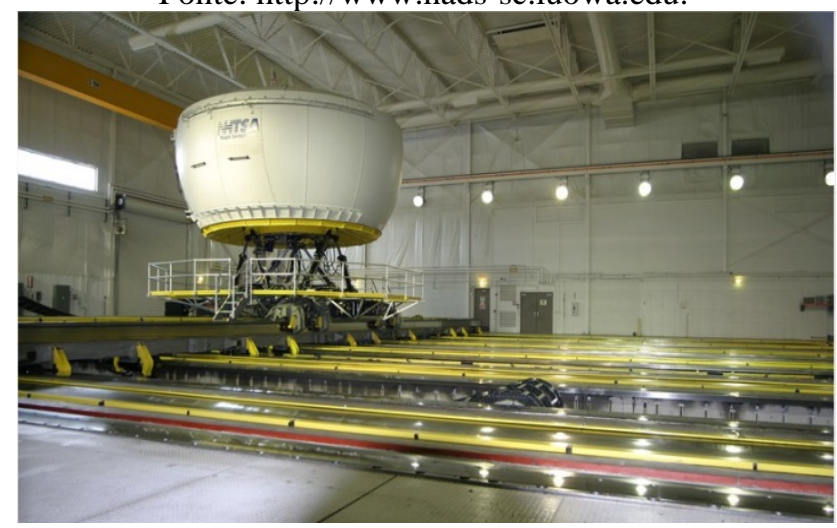

\section{APLICAÇÃO DOS SIMULADORES NA ENGENHARIA DE TRANSPORTES}

Os simuladores supracitados encontram aplicação clara no projeto de segurança de uma via. Esse projeto envolve, por sua vez, principalmente os projetos de geometria e de sinalização, porém outras áreas também podem estar correlacionadas, como por exemplo: a de estruturas, de drenagem, geotécnica, pavimentação entre outras.

Define-se geometria de uma via como os aspectos físicos da mesma, os quais incluem tópicos como alinhamento horizontal, vertical e curvatura, largura e rampas das pistas, de acordo com Granda et al. (2011). Estão associados elementos como distância de visibilidade, sinalização de segurança, de gestão do tráfego, paisagismo e iluminação (American Association of State Highway and Transportation Officials, 2001, apud GRANDA et al., 2011).

Idealmente, todas essas características da via deveriam induzir o condutor a trafegar com segurança (KEITH et al., 2005, apud GRANDA et al., 2011). Como isso não acontece na realidade torna-se relevante o papel dos simuladores no entendimento do comportamento do motorista, e consequentemente no projeto de novas vias ou adequação de vias existentes, com a principal finalidade de reduzir acidentes e fatalidades.

GRANDA et al. (2011) ressalta três perguntas fundamentais a serem feitas na consideração do uso de um simulador para estudo do projeto de via:

- Qual tipo de simulador é necessário e quais os requisitos técnicos e funcionais;

- Quais são os exemplos de pesquisas aplicadas de avaliação de projetos geométricos com o uso de simuladores;

- Quais os possíveis problemas que devem ser considerados no uso dos simuladores para tal intuito.

Bol. Ciênc. Geod., sec. Comunicações/Trab. Tecnicos Curitiba, v. 19, no 2, p.341-352, abr-jun, 2013. 


\subsection{Tipos de simuladores em função do estudo}

Conforme mencionado, os simuladores dividem-se em três níveis gerais de complexidade: básico, intermediário e de alta fidelidade. Mesmo dentro das áreas de geometria e sinalização, as possíveis pesquisas podem ser bastante diferentes entre si. Na Tabela 1 é possível identificar as pesquisas mais comuns com relação ao nível de complexidade do simulador.

Tabela 1 - Tipo de simulador mais recomendado em função do estudo. Fonte: adaptado de Granda et al. (2011).

\begin{tabular}{c|c|c|c}
\hline Pesquisa a realizar & $\begin{array}{c}\text { Nível } \\
\text { básico }\end{array}$ & $\begin{array}{c}\text { Nível } \\
\text { intermediário }\end{array}$ & $\begin{array}{c}\text { Simulador } \\
\text { de alta } \\
\text { fidelidade }\end{array}$ \\
\hline $\begin{array}{c}\text { Avaliação do projeto } \\
\text { geométrico }\end{array}$ & $\mathrm{X}$ & & \\
\hline Sinalização & $\mathrm{X}$ & & \\
\hline Geometria de via avançada & & $\mathrm{X}$ & $\mathrm{X}$ \\
\hline $\begin{array}{c}\text { Visibilidade e compreensão da } \\
\text { sinalização }\end{array}$ & & $\mathrm{X}$ & $\mathrm{X}$ \\
\hline Projeto geométrico complexo & & $\mathrm{X}$ & $\mathrm{X}$ \\
\hline $\begin{array}{c}\text { Novo projeto geométrico, } \\
\text { novas concepções }\end{array}$ & & & $\mathrm{X}$ \\
\hline $\begin{array}{c}\text { Estudos de visibilidade } \\
\text { noturna }\end{array}$ & & & \\
\hline
\end{tabular}

\subsection{Vantagens e desvantagens}

O uso de simuladores apresenta as seguintes vantagens ao avaliar o comportamento do motorista na via:

- Maior controle por parte dos pesquisadores;

- Avaliação de vias únicas ou inexistentes;

- Avaliação de medidas de segurança que seriam mais caras e/ou menos seguras de testar nas vias reais;

- Os projetistas podem visualizar e testar alternativas com antecedência;

- Cenários podem ser reutilizados em novas pesquisas.

Verifica-se entre as desvantagens, que:

- Pode ser difícil simular com fidedignidade a dinâmica do veículo;

- Desenvolver o cenário pode ser custoso;

- Geralmente os resultados precisam ser validados numa via real;

- Podem ocorrer enjoos no uso do simulador, por parte dos voluntários. 
3.2.1 Validação dos resultados

Como os simuladores são, essencialmente, instrumentos de medição, deve ocorrer uma validação dos resultados gerados (Granda et al., 2011). Nesse ínterim, existem dois tipos de validade de resultados: absoluta e relativa.

A validade absoluta significa que os resultados encontrados no simulador são iguais ou virtualmente iguais aos obtidos no mundo real. Granda et al. (2011) ressaltam que desconhecem estudos em que esse tipo de validade tenha sido reconhecida.

A validade relativa define que os resultados em campo e no simulador são diferentes, porém apresentam consistência. Por exemplo, aplicando um fator de escala, ou encontrando correspondências entre as duas aplicações.

\section{CONSIDERAÇÕES FINAIS}

O uso de simuladores de direção tem se tornado uma importante ferramenta para o desenvolvimento de estudos relativos a avaliação de aspectos de projeto que influenciam na segurança viária, principalmente em nível internacional. Sua aplicação torna possível a emulação funcional de um cenário real, sem o risco de acidentes e/ou lesões aos condutores e com custos diminuídos, o que se configura como uma grande vantagem em relação aos testes em campo - por mais que surjam outros empecilhos, como a dificuldade de elaborar modelos digitais e a necessidade de validar resultados.

A aplicação do simulador de direção permite avaliar diversas alternativas de projeto geométrico e de sinalização antes de sua implantação. Com isso, é possível efetuar uma análise comparativa das alternativas, resultando na escolha daquela que permita um tráfego mais seguro pela via, além de gerar economia de tempo e custos nas fases de projeto e execução das obras, diminuindo-se retrabalhos com a identificação de erros previamente.

Entende-se que o uso de simuladores de direção, principalmente durante a fase de concepção do projeto viário, pode contribuir fortemente para a melhoria dos aspectos de segurança e otimização dos custos. Para isso, é necessário um maior desenvolvimento de estudos e pesquisas relativos ao tema em território nacional (tanto em meio acadêmico como nas empresas de projeto) que usem como referência as linhas de pesquisa já desenvolvidas internacionalmente, adaptadas às condições e necessidades locais.

No Brasil, um passo importante está em vias de se concretizar agora, em 2013, quando os simuladores de direção deverão ser utilizados em Auto Escolas, para treinamento de condutores - Portaria 808/2012 do DENATRAN.

Entretanto, é preciso dar um passo maior, que se propõe a partir do desenvolvimento deste equipamento, para aplicação nas pesquisas voltadas para melhoria da segurança nas rodovias, na elaboração do projeto geométrico e estudos sobre comportamento dos condutores no tráfego; de forma geral, para ações de prevenção e mitigação de acidentes. 
O momento torna-se propício para este passo, dado o lançamento da chamada “Década de Ação para a Segurança no Trânsito 2011-2020”, lançada em 11 de maio de 2011 pelas Nações Unidas. A sua criação ocorreu a partir de deliberações da Assembleia Geral das Nações Unidas e Organização Mundial da Saúde exigindo que os Estados Membros priorizassem a segurança rodoviária como um assunto de desenvolvimento e saúde pública e tomassem as medidas efetivas para a redução dos números crescentes de acidentes em todo o mundo (http://www.decadatransitoseguro.com.br).

\section{AGRADECIMENTOS}

Os autores agradecem ao CNPq, pela aprovação de Projeto Universal de 2010 $n^{0}$. 470075/2010-4, através do qual foi possível iniciar esta pesquisa e a Bolsa de Produtividade $n^{0}$. 300446/2010-1; a ARTERIS - OHL BRASIL e ANTT - Agência Nacional de Transportes Terrestre - pela aprovação do projeto que permitirá o desenvolvimento de simulador realístico de direção no Departamento de Engenharia de Transportes da Escola de Engenharia de São Carlos, em parceria com o Departamento de Engenharia de Transportes da Escola Politécnica, ambos da Universidade de São Paulo e finalmente a FAPESP pela bolsa para participação no evento $16^{\text {th }}$ International Conference Road Safety on Four Continents, em Beijing, China, em maio de 2013, para atualização do estado-da-arte das pesquisas em segurança de rodovias.

\section{REFERÊNCIAS BIBLIOGRÁFICAS}

ALLEN, R. W; Park, G. D.; Joe, W. S.; Goddard, P. A. (2008). Hardware-in-theLoop Simulation of Braking Capability. Presented at DSC2008 Driving Simulator Conference and Exhibition, Monaco, 31 January-1 February 2008.

ANDERSEN, G. J. (2011). Sensory and Perceptual Factors in the Design of Driving Simulation Displays. In: FISHER, D. L.; RIZZO, M.; CAIRD, J. K.; LEE, J. D. Handbook of Driving Simulation for Engineering, Medicine, and Psychology. CRC Press, Taylor \& Francis Group. P.8-1. 2011.

BELLA, F. (2008). "Driving Simulator for Speed Research on Two-Lane Rural Roads”, Accident Analysis and Prevention, 40, 1078-1087.

BENEDETTO, A., DE ANGELINI, A., DI RENZO, D., GUERRIERI, F., AND MARKHAM, S. (2002). "About the Standards of a Driving Simulation for Road Engineering: A New Approach”, Proceedings of the Seventh International Conference Applications of Advanced Technologies in Transportation ASCE, 704-711.

BHATTI, G.; JESSEL, JP.; BREMOND, R.; MILLET, G.; VIENNE, F. (2012). Towards the development of a user interface to model scenarios for driving simulations. Driving Simulation Conference 2012 - Europe. Paris, França.

CÂMARA DO DEPUTADOS, divisão Transporte e Transito (2012). http://www2.camara.leg.br/camaranoticias/noticias/TRANSPORTE-E- 
TRANSITO/426865-SEMANA-NACIONAL-DE-TRANSITO-ALERTAPARA-NECESSIDADE-DE-REDUZIR-ACIDENTES-NO-PAIS.html

CHO, J. H; KIM, Y. S.; SOHN, Y. W.; KNOW, J. Y.; PARK, S. A.; LEE, K. S.; CHUNG, E. K.; SOHN, H. K. (2011). Development of a Driving Simulator for the Driving Precision Aptitude Test. Proceedings of $3^{\text {rd }}$ International Conference on Road Safety and Simulation. Indiana Government Conference Center Indianapolis. September, Indiana, Indiana, EUA. 2011.

COMITÊ 16TH INTERNATIONAL CONFERENCE ROAD SAFETY ON FOUR CONTINENTS (2013). http://www.vti.se/en/road-safety-on-four-continents/

FISCHER, M.; ERIKSSON, L. (2012). Swedish National Road and Transport Research Institute (VTI), (Sweden) Evaluation of methods for measuring speed perception in a driving simulator. Driving Simulation Conference 2012 Europe. Paris, França.

GODLEY, S.T., TRIGGS, T.J., AND FILDES, B.N. (2002). "Driving Simulator Validation for Speed Research”, Accident Analysis and Prevention, 34, 589600.

GRANDA, T. M.; DAVIS, D. W.; INMAN, V. W.; MOLINO, J. A. (2011). Cap. 34 - The Use of High-Fidelity Real-Time Driving Simulators for Geometric Design. Handbook of Driving Simulation for Engineering, Medicine and Psychology. CRC Press, 2011.

HONG, I. K.; RYU, J. B.; CHO, J. H.; LEE, K. H.; LEE, W. S. (2011). Development of a Driving Simulator for Virtual Experience and Training of Drunk Driving. Proceedings of $3^{\text {rd }}$ International Conference on Road Safety and Simulation. Indiana Government Conference Center Indianapolis. September, Indiana, EUA. 2011.

JAMSON, H. (2011). Cross-Plataform Validation Issues. In: FISHER, D. L.; RIZZO, M.; CAIRD, J. K.; LEE, J. D. Handbook of Driving Simulation for Engineering, Medicine, and Psychology. CRC Press, Taylor \& Francis Group. p. 12-10. 2011.

KEARNEY, J. K.; GRECHKIN, T. F. Selecting a Driving Simulator. In: FISHER, D. L.; RIZZO, M.; CAIRD, J. K.; LEE, J. D. (2011). Handbook of Driving Simulation for Engineering, Medicine, and Psychology. CRC Press, Taylor \& Francis Group. P 6-1. 2011.

KLEE, H., BAUER, C., RADWAN, E., AND AL-DEEK, H. (1999). "Preliminary Validation of Driving Simulator Based on Forward Speed”, Transportation Research Record, 1689, 33-39.

KUMAR, S. N.; UMADEVI, G. (2011). Application of System Dynamic Simulation Modeling in Road Safety. Proceedings of $3^{\text {rd }}$ International Conference on Road Safety and Simulation. Indiana Government Conference Center Indianapolis. September, 2011, Indiana, EUA.

OLIVER, J. (2012). Driving simulation applications in immersive environments. Driving Simulation Conference 2012 - Europe. Paris, França. 
SANTIAGO-CHAPARRO, K., DEAMICO, M., BILL, A., CHITTURI, M., NOYCE, D. (2011). Virtual Road Safety Audits Using Driving Simulators: A Framework. $3^{\text {rd }}$ International Conference on Road Safety and Simulation. Indianápolis, Indiana, EUA.

UNIVERSITY OF LEEDS DRIVING SIMULATOR (2012). Disponível em: <www.its.leeds.ac.uk>. Acesso em 14 nov. 2012.

(Recebido em janeiro de 2013. Aceito em março de 2013). 